\title{
Performance Analysis of a Haptic Telemanipulation Task under Time Delay
}

\author{
Michael C. Yip ${ }^{\mathrm{a}, *}$, Mahdi Tavakoli ${ }^{\mathrm{b}}$ and Robert D. Howe ${ }^{\mathrm{c}}$ \\ ${ }^{a}$ Department of Electrical and Computer Engineering, University of British Columbia, \\ 2332 Main Mall, Vancouver, BC, Canada V6T 1 Z4 \\ ${ }^{\mathrm{b}}$ Department of Electrical and Computer Engineering, University of Alberta, Edmonton, AB, \\ Canada T6G 2V4 \\ ${ }^{\mathrm{c}}$ School of Engineering and Applied Sciences, Harvard University, Cambridge, MA 02138, USA
}

Received 7 May 2010; accepted 6 July 2010

\begin{abstract}
There is ample research on the effect of haptic teleoperation under delayed communication channels in terms of stability and system performance. Little attention, however, has been paid to the effect of delayed force feedback on users' task performance and whether force feedback is beneficial under significant communication delays. This paper investigates whether force feedback improves user's task performance in delayed teleoperation. We study peg-in-the-hole insertion/retraction, dexterous manipulation tasks involving high degrees of freedom and high forces at certain points during task execution. A user study involving unilateral (without force feedback), bilateral (with force feedback) and graphical feedback teleoperation under various delays is presented. We observed that for all feedback modalities, task completion times increase as delay increases. Haptic feedback helps reduce contact forces and the occurrence of large robot/environment forces. Furthermore, graphical feedback helps users maintain the lowest range of forces at the cost of higher task completion times. With users mindful of minimizing contact forces, haptic/graphical feedback causes the task to take more time than unilateral control. Therefore, when short completion times are crucial given a tolerance for larger forces, force feedback only serves to increase the time required to perform the task; thus, unilateral control may be sufficient.
\end{abstract}

(C) Koninklijke Brill NV, Leiden and The Robotics Society of Japan, 2011

\section{Keywords}

Haptic teleoperation, task performance, manipulation task, delayed teleoperation

\section{Introduction}

As a human operator controls the position of a teleoperated robot through a user interface and performs a task such as grasping an object in a remote environment,

\footnotetext{
* To whom correspondence should be addressed. E-mail: myip@ece.ubc.ca
} 
capturing the robot-object contact forces and bringing them to the operator in the form of haptic (force) feedback will engage the operator's sense of touch and may enable better control over the contact forces. The main goals of such a haptic teleoperation system are stability and transparency, which is defined as the ability to present undistorted dynamics of the remote environment to the human operator [1, 2]. An interesting control problem arises from the presence of a non-negligible time delay in the communication media between the user interface (master) and the teleoperated robot (slave), which severely affects the stability and transparency of the haptic teleoperation system. Several approaches have been proposed in the literature to deal with this problem. For a comprehensive overview and comparison of various delay compensation methods, see Refs [3-6].

The passivity-based approach to delay compensation, which has been expressed in the scattering framework [7] and in the wave transformation framework [8], is an efficient method for stabilizing a teleoperation system independent of transmission delays. The key to this approach is making the non-passive delayed communication medium passive (at the expense of transparency degradation). This approach does not eliminate or reduce the delay; thus, the potentially harmful effects of delay on performance persist.

While there is ample research on delayed teleoperation system stabilization, little attention has been paid to the effect of delayed haptic feedback on task performance, i.e., whether, and for what class of tasks, haptic interaction actually helps to improve task performance in delayed teleoperation. While the usefulness of haptic feedback in no-delay teleoperation has been established, e.g., in Refs [9-11], the loss of temporal coincidence between the human operator's motions and the ensuing reflected forces in delayed teleoperation may confuse the operator so much so that the force feedback becomes useless or even misleading. Thus, this paper aims to study how delayed haptic feedback affects the user task performance in a typical manipulation task.

To assess the value of providing haptic feedback to the user during delayed teleoperation, prior work studied the effect of delay on the human's perception of the relative stiffness of virtual spring-like surfaces simulated by reflecting forces proportional to the user's virtual surface indentations. Subjected to a forced-choice paradigm (i.e., distinguish the stiffer of the two surfaces or identify them as having the same stiffness), users perceived the surfaces to be stiffer than actual under delayed force feedback and the stiffness overestimation increased for larger delays $[12,13]$. Recently, the effect of crossing the boundary of a force field, where local stiffness is ill-defined, on the perception of delayed stiffness has been studied [14]. It was found that subjects interacting with delayed force fields underestimate (overestimate) stiffness if they do not move (do move) across the boundary of the elastic field.

While the literature has so far been limited to studying the stiffness discrimination task, we consider the effect of delayed haptic feedback on the performance of a peg-in-the-hole insertion task. The differences are in that stiffness discrimination 
is a single-degree-of-freedom, low-force, sensing task while peg-in-the-hole insertion is a multi-degree-of-freedom, both low- and high-force, manipulation task. The complexities of this dexterous manipulation task are likely to affect the value of haptic feedback in the presence of delay.

It is noteworthy that, for the case of no-delay teleoperation, haptic feedback has been shown to help task performance in different ways depending on the levels of forces [11]. At high levels of force feedback, environment mechanical properties are presented against the user's hand as passive physical constraints, which serve both as safety barriers and as intuitive guides for tools. At low levels of force feedback, however, force feedback is beneficial less as a physical constraint and more as a supplemental information source requiring an increased level of awareness and cognitive processing by the user. Such a difference in the underlying mechanisms of haptic assistance motivated us to consider the peg-in-the-hole insertion task as it requires the user to exert high levels of force at some points during the operation and coordinate low levels of force at some other points during the task execution.

A general hypothesis supported by past work [12-14] is that time delays in position feedforward and haptic feedback can disturb the human operator and cause misjudgement. The key question we pose in this paper is, how is the performance of this basic manipulation task influenced by delays in haptic feedback? This work should not be confused with prior work analytically or empirically studying how wave transformations limit the performance of teleoperation systems due to displaying distorted inertia and damping to the operator, the wave reflection phenomenon, etc. Instead of system performance, we directly study task performance (user performance) under delayed haptic feedback through teleoperation experiments performed by a pool of human subjects.

On the other hand, in teleoperation applications with large time delays, such as ground-to-space teleoperation where it is difficult to compensate for the adverse effect of the large delay on haptic teleoperation system stability and performance, graphical substitution for haptic feedback is a viable alternative to the haptic feedback. Similarly, if the user interface has a force reflection capability in fewer degrees of freedom than those of the task, partial force reflection may destabilize the teleoperation system [15], in which case replacing haptic feedback by graphical feedback is useful. Such graphical 'sensory substitution' has been found to improve a user's sensitivity for detecting small forces by allowing the use of high feedback gains without slowing down hand movements [16]. For manual and telerobotics operations of a surgical knot tying task, the forces applied in the sensory-substitution mode were closer to the forces applied in the manual mode when the users were provided with auditory/graphical representation of haptic information [17]. It would be interesting to see the difference between haptic feedback and graphical haptic feedback in the robotic mode itself. In this paper, the two contact feedback modalities are compared in terms of their capability in transmitting critical task-related information to the user. 
The main contribution of the paper is studying the combined effects of time delay and feedback modality (unilateral or without force feedback, bilateral or with force feedback and graphical feedback) on task performance. It must be noted that past user studies have looked at either the effect of haptic feedback on task performance in the absence of delay or at the effect of various delays on task performance in the absence of haptic feedback (i.e., one-dimensional comparisons). In this paper, for the first time, a two-dimensional comparison of task performance is carried out across both various modalities and various delays.

This paper is organized as follows. In Section 2, we describe the experimental setup including the teleoperation system and how it is stabilized under time delay. Section 3 describes the experimental protocol when users executed a peg insertion task under various delays, and explains how the task was segmented and evaluated in terms of performance. Section 4 presents the results including the effect of delay on task completion time and the effect of feedback modality on energy transfer to the environment. Section 5 discusses and compares the results, and Section 6 includes concluding remarks. An overview of the $t$-test and analysis of variance (ANOVA) is given in the Appendix.

\section{Experimental Setup}

Two 6-d.o.f. PHANToM Premium 1.5 devices (SensAble Technologies, Woburn, MA, USA) were used, one as the master device and one as the slave device. Figure 1 shows the experimental setup. On the operator end, the user manipulates the master PHANToM robot and the motion commands are transmitted to the slave robot through a delayed communication channel. On the slave side, a cylindrical peg made of aluminum is attached to the endpoint of the second PHANToM robot, which is placed next to an aluminum block with a cylindrical hole that offers a sliding fit with the peg. The interaction forces between the peg and the hole (i.e., the slave/environment contact forces) are transmitted back to the master side through a

(a)

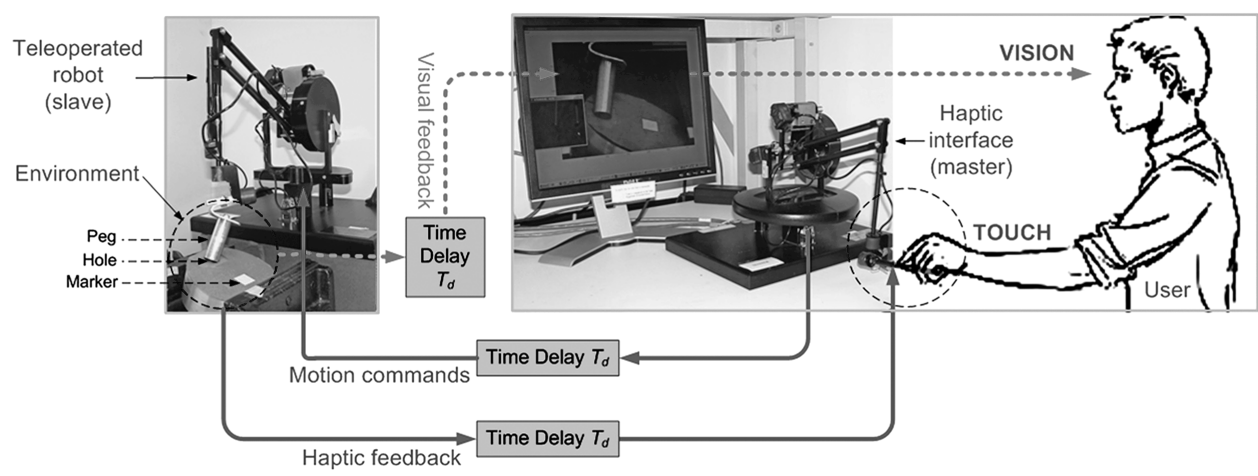

Figure 1. Teleoperated robot and peg/hole setup (a), and the user's haptic/visual interface (b). 
Table 1.

Peg-in-the-hole setup parameters

\begin{tabular}{lll}
\hline Parameter & Symbol & Value \\
\hline Peg diameter & $d_{\mathrm{H}}$ & $18.38 \mathrm{~mm}$ \\
Hole diameter & $d_{\mathrm{P}}$ & $18.60 \mathrm{~mm}$ \\
Peg height & $h$ & $52 \mathrm{~mm}$ \\
Hole tilt angle & $\theta$ & $21^{\circ}$ \\
\hline
\end{tabular}

similarly delayed communication channel. A video feed aimed at the slave-side environment (the peg and the hole) provides visual feedback of interactions between the slave and the environment to the operator, and is purposely designed to suffer from the same communication channel delay as the force feedback. The position and force sampling rate on both master and slave devices is $1 \mathrm{kHz}$.

The peg-in-the-hole task is a common manipulation task that requires multiple degrees of freedom. Peg-in-the-hole setups are generally characterized using a precision value defined as:

$$
I=\log _{2}\left(\frac{d_{\mathrm{H}}}{d_{\mathrm{H}}-d_{\mathrm{P}}}\right),
$$

where $d_{\mathrm{H}}$ is the diameter of the hole and $d_{\mathrm{P}}$ is the diameter of the peg. Table 1 lists the parameters of our peg-in-the-hole setup; the precision value of our setup is $I=1.922$. Other studies have used precision values within the same order of magnitude [18].

A controller for stabilizing the master-slave system under time-delayed communication was required for the case of bilateral teleoperation. The passivity-based wave variable method by Anderson and Spong [7] was used as it is the most popular and well-studied method for ensuring a passivated communication channel between the master and the slave. A pair of wave variables $(u, v)$ is defined based on standard power variables, i.e., velocity and force $(V, F)$, according to:

$$
u=\frac{b V+F}{\sqrt{2 b}}, \quad v=\frac{b V-F}{\sqrt{2 b}},
$$

where $b$ is called the characteristic wave impedance and is a positive constant that must be tuned in order to tradeoff the speed of motion for the levels of force feedback [8]. In general, when the characteristic wave impedance is decreased (increased), force levels are lower (higher) and the system shows less (more) damping to the operator. In our experimental setup, the characteristic wave impedance was tuned empirically, aiming to achieve both easy motion (low damping) when the slave is in free space and high levels of force feedback when the slave is in contact with a stiff environment. For the best speed of motion and force feedback, $b$ was set to $b=10 \times 10^{-3}$ with the exception of $b=16 \times 10^{-3}$ when the one-way delay was $500 \mathrm{~ms}$. 


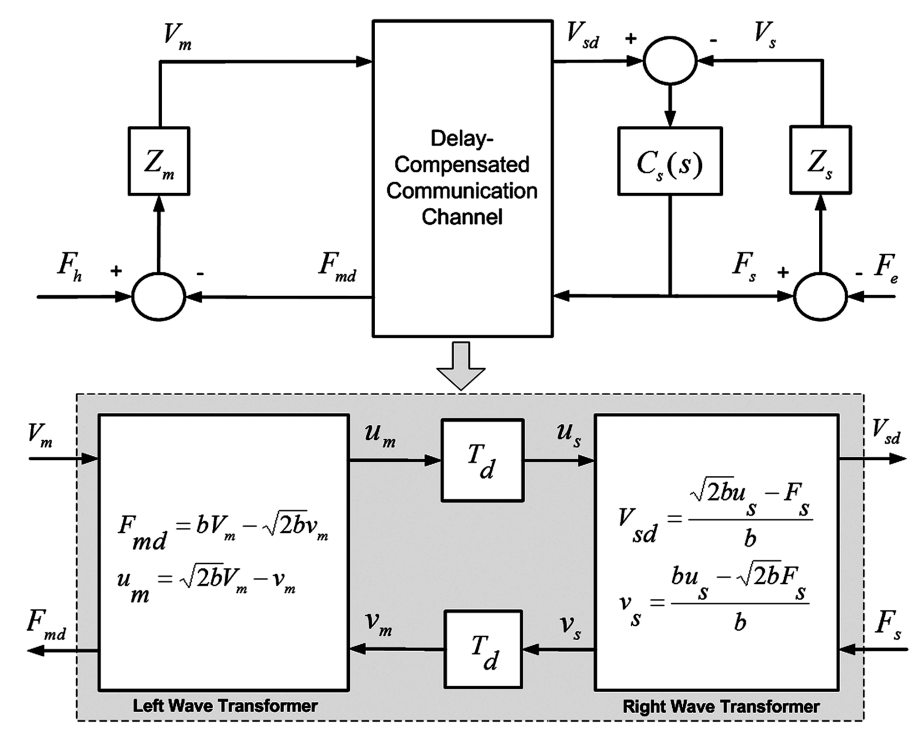

Figure 2. Passivity-based, delay-compensated PEB teleoperation system.

Interestingly, using appropriate pairs of such wave transforms at the two ends of a delayed communication channel can make it passive. Such a 'passivated' (delay-compensated) communication channel guarantees the teleoperation stability assuming that the remote environment and the operator display passive behaviors.

In this paper, the communication time delay $T_{\mathrm{d}}$ has been assumed to be constant and equal in both directions (even though the assumption of equal forward and backward delays is not necessary for ensuring passivity). The resulting control architecture encompassing the passivated communication channel and using the position error-based (PEB) controller is shown in Fig. 2. Here, $Z_{\mathrm{m}}$ and $Z_{\mathrm{s}}$ are the master and slave impedances, and $F_{\mathrm{h}}$ and $F_{\mathrm{e}}$ are the operator/master and the slave/environment interaction forces. Also, $V_{\mathrm{m}}$ and $V_{\mathrm{s}}$ are the master and the slave robots velocities in the $s$-domain. Lastly, $F_{\mathrm{m}}$ and $F_{\mathrm{s}}$ are the master and slave control actions. The PEB controller does not use any force sensor measurements; rather, it tries to minimize the difference between the (delayed) master position/velocity and the slave position/velocity, reflecting a force that is proportional to this difference to the user once the slave makes contact with an object.

As mentioned in Section 1, prior research [15-17] provided motivation to consider alternate methods of displaying the slave/environment contact forces to the operator such as graphical feedback. In our experiments, whereas haptic feedback displayed forces between the slave and the environment as a reaction force exerted by the master on the operator's hand, graphical feedback displayed the same forces in a computer monitor as three-dimension vectors in Cartesian coordinates. To do so, the reaction forces were decoupled into component forces $f_{i} \in\left\{f_{x}, f_{y}, f_{z}\right\}$ along Cartesian axes and the vectors displayed orthogonal to one another were proportional to the corresponding force component. The components of the force 

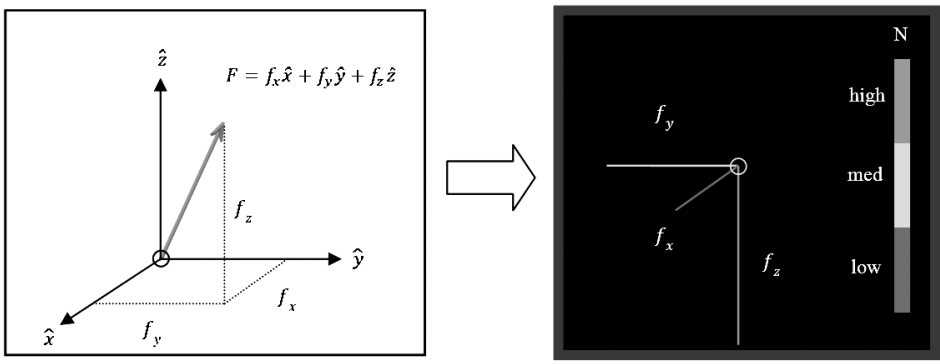

Figure 3. (Left) Reaction force $F$ from the remote environment on the slave robot with components $\left\{f_{x}, f_{y}, f_{z}\right\}$. (Right) Graphical feedback provided to the user shown in isometric projection, indicating the direction and intensity of applied forces.

vectors $\left(f_{i}\right)$ were shown to the user using different colors depending on the force range (green (low): $f_{i} \leqslant 1 \mathrm{~N}$; yellow (med): $1 \mathrm{~N}<f_{i} \leqslant 2 \mathrm{~N}$; red (high): $f_{i}>2 \mathrm{~N}$ ). This graphical feedback was delayed by the same amount as the haptic feedback and was updated at a sufficiently high rate. Figure 3 shows the user interface corresponding to the graphical feedback. To provide the most intuitive graphical feedback to the user, the reaction forces were reversed in direction to show a deviation from the origin (where forces are zero). Therefore, by moving the master in the direction from the tip of a line back to the origin, the users could reduce the applied forces.

\section{Procedure}

Various combinations of the two study variables that characterize our experiments were used to determine the test cases: teleoperation modality (unilateral, bilateral or graphical feedback) and communication delay (0, 100, 200 or $500 \mathrm{~ms})$. Communication delays up to $500 \mathrm{~ms}$ span the range typically encountered in current teleoperation systems. The delays above correspond to single-way communication and, therefore, round-trip signals experience double the delays. The video feedback of the remote environment provided to the operator lags by the same delay amount as well.

Eight adult subjects (six male and two female; average age of 28) with little past exposures to haptic feedback and graphical force feedback participated in the experiments. Peg-in-the-hole insertion under each feedback modality/delay pairing (i.e., $3 \times 4=12$ different experimental conditions) was performed twice by each subject, (i.e., 24 trials for each experimental condition and the total number of trials $N=8 \times 24=192$ ). The subjects were asked at the beginning of experiments to minimize the levels of contact forces, as well as the task completion times. Each subject was given a few practice trials at the beginning under the different experimental conditions until he or she was comfortable with the operation of the master-slave system, the different feedback modalities and the execution of the peg-in-hole task. For the experiment, the modality/delay pairs were presented to the subjects in a randomized order to minimize the effects of learning. The subjects 
were informed of the amount of delay for each trial. The master and slave positions and the task completion time were recorded for each trial, and the interaction forces were determined from the PEB-based controller (in which forces are proportional to the difference between master and slave positions/velocities).

\subsection{Task Sequence and Segmentation}

A peg-in-the-hole run began with the peg located inside the hole, and consisted of the following four operations (subtasks):

(i) Retract the peg from the hole.

(ii) Move to a distant position, tap a marker, return the peg to the hole entrance.

(iii) Align the peg with the hole.

(iv) Insert the peg into the hole.

The data was segmented based on the radial distances of the peg from three points: a starting location (determined when the peg is fully inside the hole), a distant location (a marked position about $40 \mathrm{~mm}$ far from the hole) and an alignment location (determined when the peg is aligned prior to insertion). When the trajectory of the peg leaves one point's radial vicinity, it was considered as entering a new subtask. Out of the four subtasks listed above, we were only concerned about the retraction (i), alignment (iii) and insertion (iv) subtasks. For subtask (ii), users were not required to tap the marker with accuracy; rather, it simply served as a separation point between the peg retraction and the peg alignment subtasks. By requiring users to reposition and realign the peg during the alignment subtasks, we ensured that the alignment of the peg was not assisted by its previous position and orientation after retraction. A sample trajectory and its automated segmentation into its various subtasks are shown in Fig. 4.

\subsection{Performance Measures}

We considered the following metrics to evaluate the performance of the task under different experimental conditions:

- Completion time. Completion times were saved for all relevant subtasks (extraction, alignment and insertion) as well as for the entire task.

- Sum of squared forces (SOSF) and average sum of squared forces (ASOSF). The $S O S F$ was used to give an absolute measure of the force interactions between the slave and the environment, and was defined as:

$$
S O S F=\sum_{i=1}^{n}|F|^{2},
$$



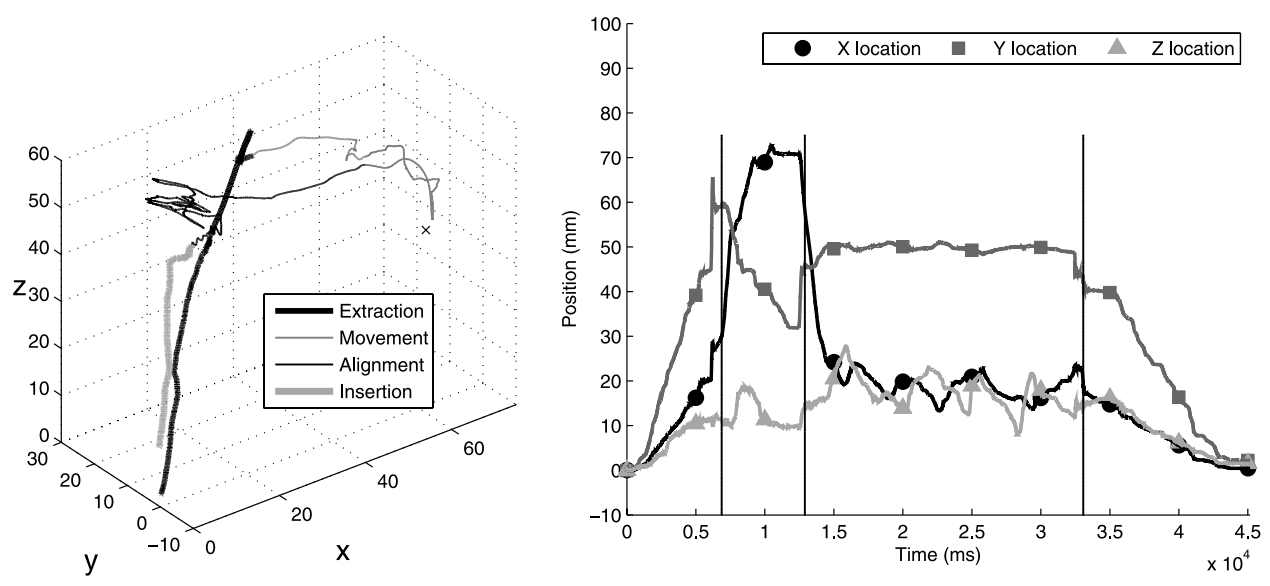

Figure 4. Sample trajectory of the peg and the segmentation of different subtasks: The three-dimensional trajectory (left) and the one-dimensional trajectories in $x, y$ and $z$ directions (right).

where $|F|$ is the absolute force magnitude and $n$ is the number of captured data samples in the subtask of interest. We were also interested in the ASOSF:

$$
A S O S F=\frac{1}{n} \sum_{i=1}^{n}|F|^{2},
$$

as it gives an indication of the rate at which forces are being applied to the environment. Both SOSF and ASOSF can also be considered to measure the amount and rate of effort required by the human operator to perform the task.

- Force histograms. Force histograms give a picture of the frequency of occurrence of different force levels. An absolute force histogram plot is scaled by the completion time, whereas normalizing force histograms over time (whereby the measured variable is a percentage of time exerting a certain force) allows one to compare the average forces of different feedback modality/delay pairings.

- 95th percentile forces. The 95th percentile force were considered to represent the overall maximum force (because $95 \%$ of the time the force is below this amount). Considering these maximum forces gives insight into how 'rough' the interactions are between the teleoperated robot and its environment.

\section{Results}

The following section compares the results based on the six performance measures mentioned above: completion time, $S O S F, A S O S F$, force histograms, normalized force histograms and 95th percentile forces. With the exception of the force histograms, both standard errors (SEs) of the means and standard deviations (SDs) are 
provided for all performance measures. Let $x$ be a random variable of $N$ samples with mean $\bar{x}$. The SD, defined as

$$
\mathrm{SD}=\sqrt{\frac{1}{N-1} \sum_{i=1}^{N}\left(x_{i}-\bar{x}\right)^{2}},
$$

will help understand the variance of the performance measures from trial to trial and from subject to subject, whereas the SE, defined as

$$
\mathrm{SE}=\frac{\mathrm{SD}}{\sqrt{N}}
$$

will provide an indication of our ability to estimate the mean value of a performance measure.

\subsection{Completion Time}

Figure 5 shows the mean time and the standard error required to complete each subtask under different delays. Regardless of the teleoperation modality, the task completion times for all subtasks can be seen to increase as delay increases, with overall task completion times increasing from 14.3 to $34.1 \mathrm{~s}$ (unilateral), from 25.5 to $54.2 \mathrm{~s}$ (graphical) and from 18.9 to $39.7 \mathrm{~s}$ (bilateral) as the delay increases from 0 to $500 \mathrm{~ms}$. When the users are given haptic feedback, the completion times increase by about $5 \mathrm{~s}$ compared to the unilateral mode for all delays levels. Furthermore, the use of graphical mode compared to the unilateral mode increases the task completion times anywhere from approximately 10 to $20 \mathrm{~s}$.

In order to determine the statistical significance of these results, a two-tailed Student's $t$-test (see the Appendix) was performed to determine whether there is a discernable difference in the mean values of two feedback modalities (see Table 2).
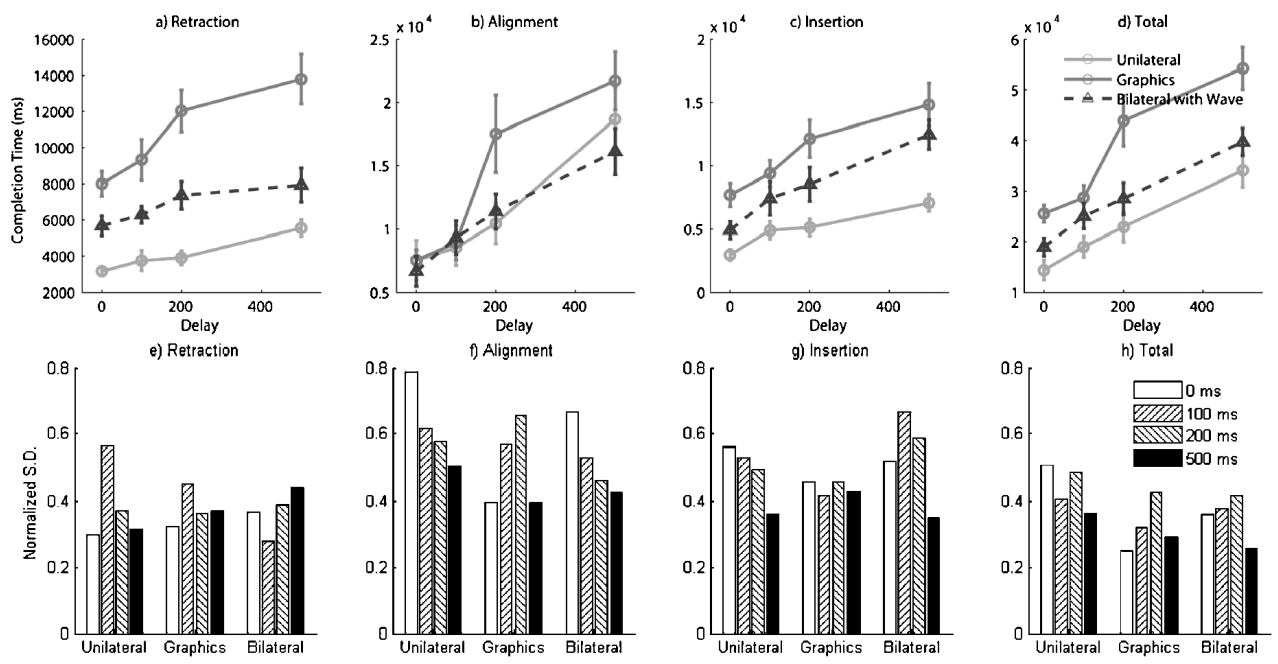

Figure 5. Task completion times across delay. Bars show the standard errors. 


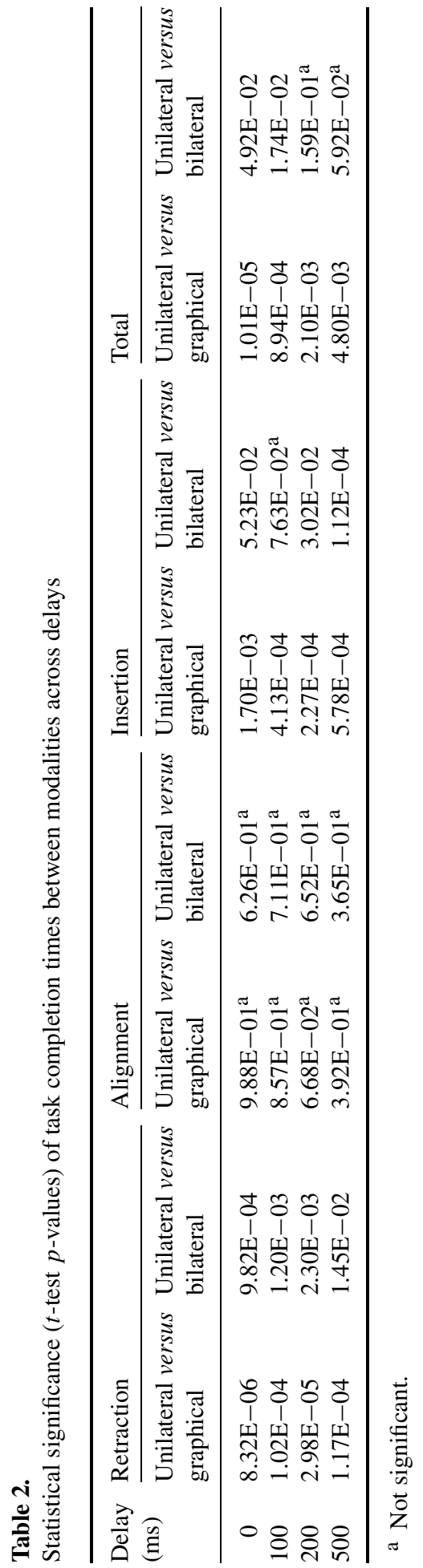


The $t$-test results confirm that both graphical feedback and bilateral control have a significant effect on the completion time of the subtask for both retraction and insertion subtasks.

The SDs in Fig. 5e-h have been normalized with the mean completion time of the specific subtask. Throughout the entire task (retraction, alignment and insertion), we do not see a significant change in the normalized standard deviations, implying that the variance in the completion times, when normalized with the total time taken for the task, generally remains the same regardless of delay.

\section{2. $\mathrm{SOSF} / \mathrm{ASOSF}$}

To get a sense of the absolute force interactions between the slave and the environment, the SOSF was calculated at each delay (see Fig. 6a-d). We performed an ANOVA to determine the statistical significance across delays (see Table 3). The results in Fig. 6a-d show that the presence of haptic feedback reduces the $S O S F$ at each delay and effectively stabilizes the total SOSF from changing significantly as the delay increases $(F=1.30 \mathrm{E}+00, p=2.83 \mathrm{E}-01)$. In contrast, the unilateral mode does increase the SOSF as the delay increases $(F=3.54 \mathrm{E}+00$, $p=2.06 \mathrm{E}-02)$, and so does the graphical feedback mode $(F=4.6141, p=$ $6.17 \mathrm{E}-03)$.

To further understand the effects being seen, we have normalized the SOSF by time in order to get the $A S O S F$ since different feedback modalities required significantly different times to complete each subtask. Figure $7 \mathrm{a}-\mathrm{d}$ shows the ASOSF, and the corresponding standard deviations for unilateral, graphical and bilateral modalities. Bilateral teleoperation maintains a nearly constant ASOSF for all delays (validated by ANOVA, Table 4: $F=646 \mathrm{E}-01, p=5.89 \mathrm{E}-01$ ). However, there is a significant change when comparing the SOSF and the ASOSF results
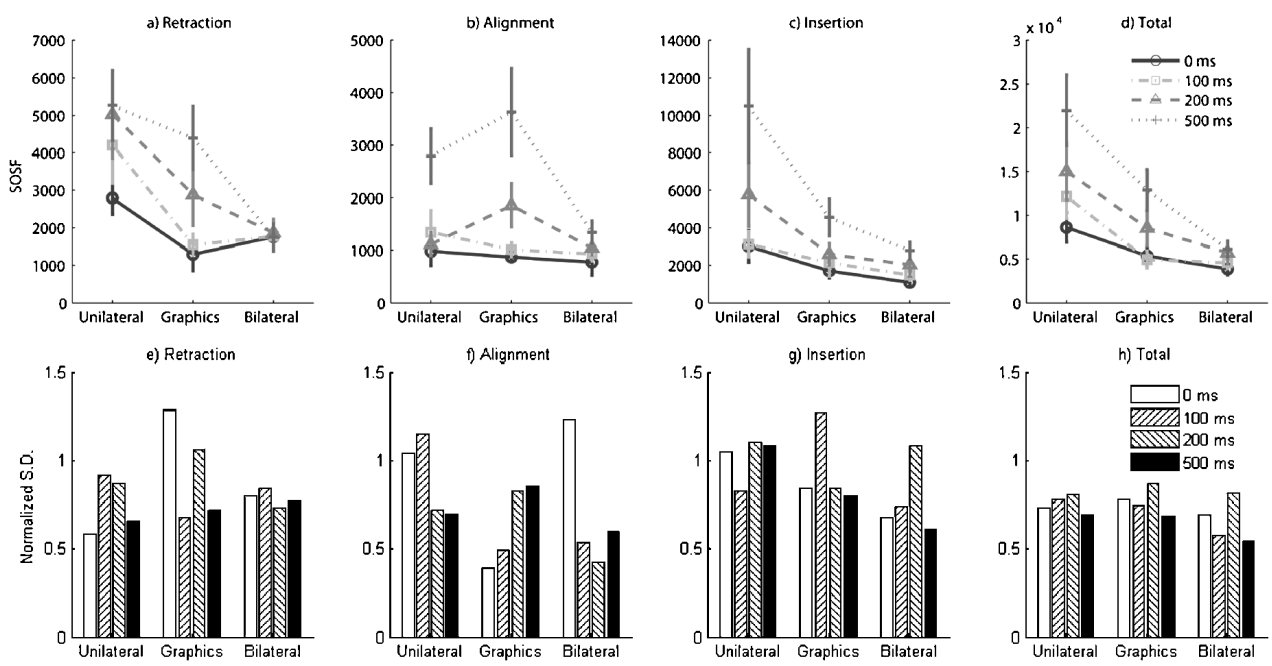

Figure 6. SOSF and corresponding SDs across modality. 


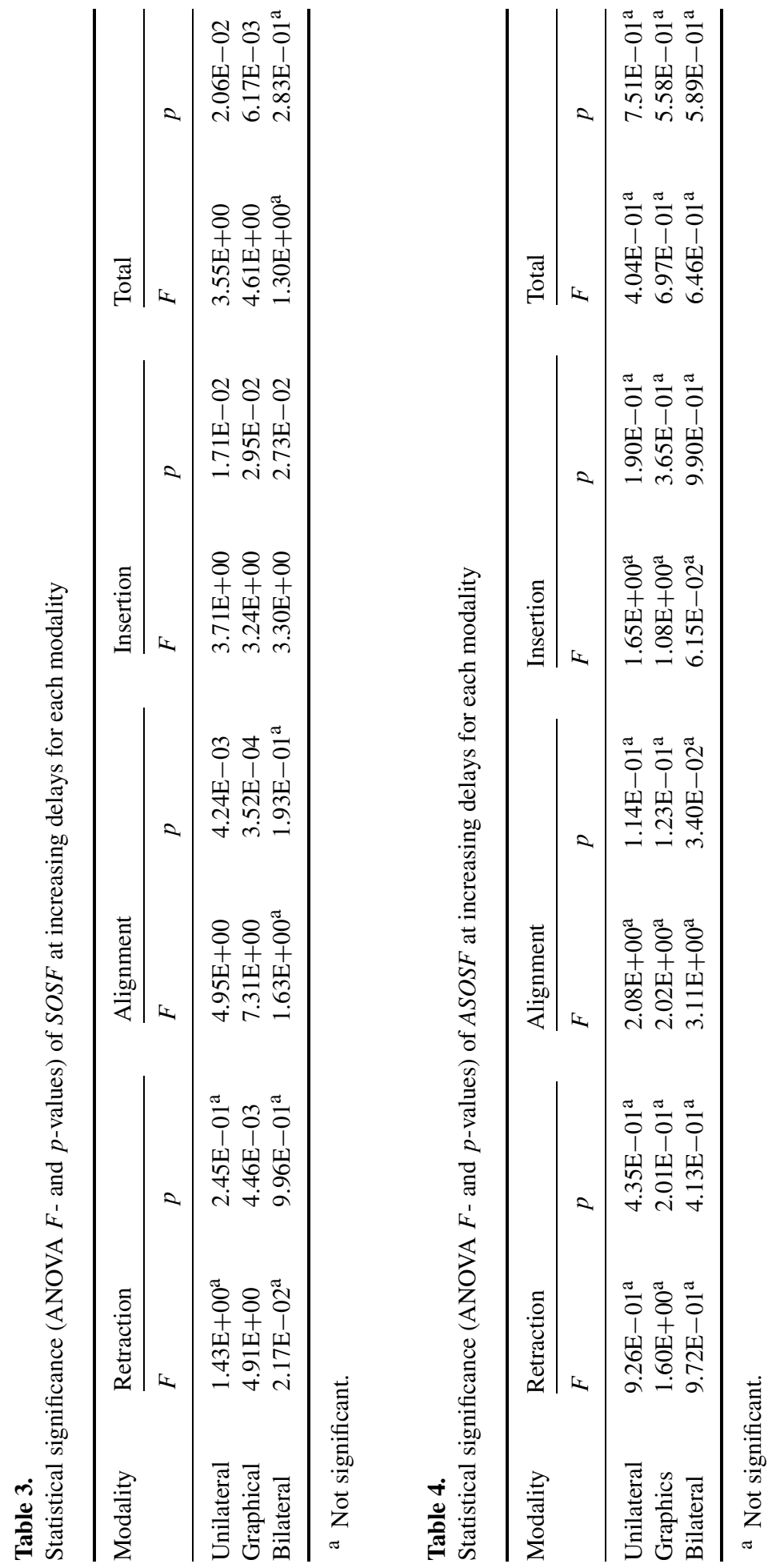



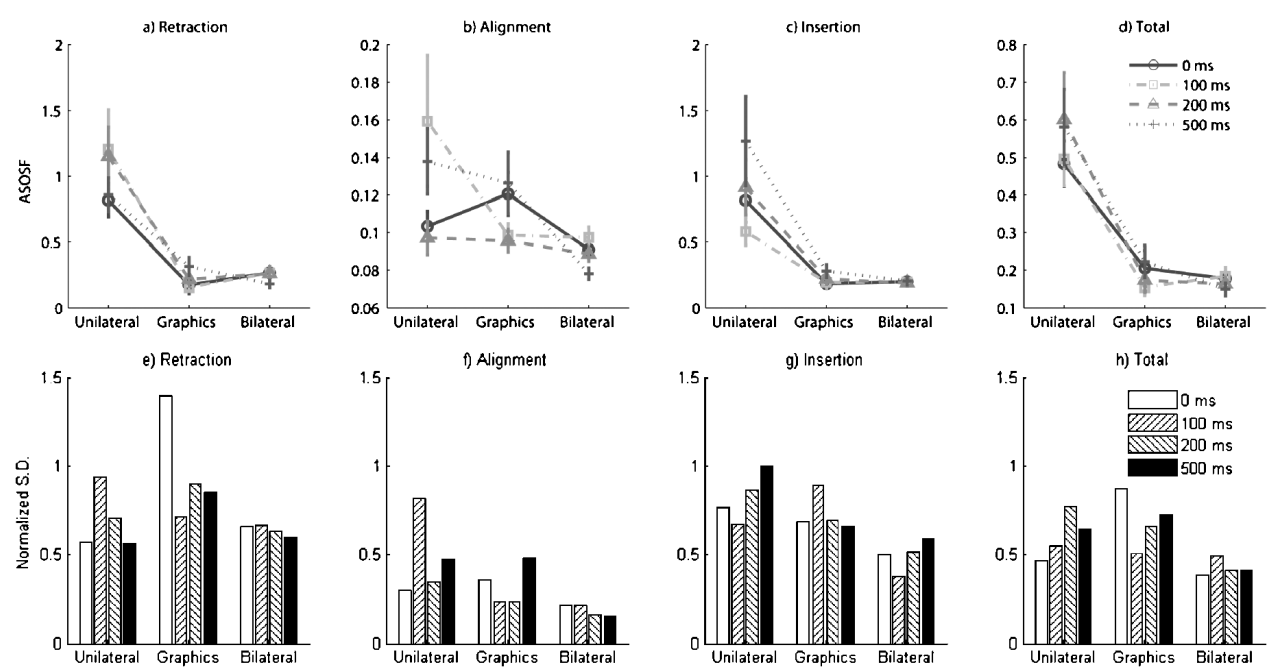

Figure 7. ASOSF and corresponding SDs across modality.

for graphical feedback. Whereas the SOSF for grahical feedback were significantly increasing as delay increased, the ASOSF for graphical feedback showed a fairly constant value regardless of delay in the system $(F=6.97 \mathrm{E}-01, p=5.58 \mathrm{E}-01)$ with values similar to bilateral mode. Therefore, these results suggest that force interactions on average are significantly smaller when the user is given graphical feedback and, despite changes in delay, these average force interactions are not changing significantly. Figure $7 \mathrm{a}-\mathrm{d}$ shows that unilateral teleoperation causes more forces on average to be exerted on the environment compared to bilateral teleoperation and graphical feedback. Similar to the graphical feedback's ASOSF, the $A S O S F$ for unilateral teleoperation does not increase as significantly across delays $(F=0.4037, p=0.7509)$.

It was also observed that the SDs for bilateral teleoperation were generally lower than for unilateral teleoperation, as well as graphical feedback teleoperation, indicating that the variance in the ASOSF is lower when the subject is provided with haptic feedback (see Fig. 7e-h).

\subsection{Force Histograms}

Absolute force distribution plots in Fig. 8 provide an understanding of the occurrences of forces (ranging from 0 to $3 \mathrm{~N}$ ) for all delays and modalities. During alignment (Fig. 8b), the addition of haptic feedback does not significantly change the force distribution. However, it is seen that bilateral control results in fewer occurrences of high force values across all delays during retraction and insertion. Unilateral mode induces large forces more often but also fewer small forces due to its faster completion time. For retraction and insertion, while the general shapes and peaks of unilateral's distributions do not significantly change as the delay in- 

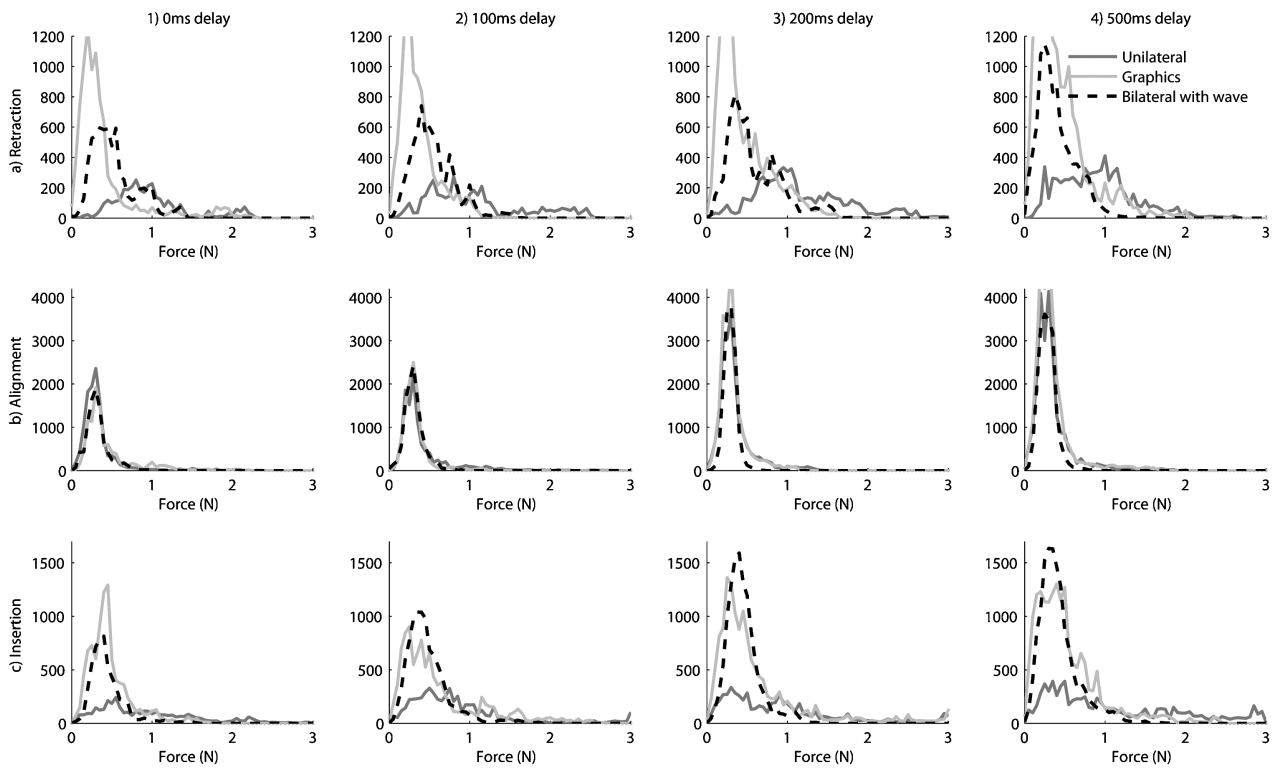

Figure 8. Force histograms across delay.

creases, the peaks of bilateral's distributions show an increase in occurrence as the delay increases (Fig. 8a and c).

We also considered the completion time-normalized force histogram distribution (in which the area beneath the curve is equal to unity) in order to compare between feedback modalities the percentage of time spent at certain forces. We see that during retraction (Fig. 9a), both graphical feedback and bilateral modalities result in lower forces on average than unilateral for all delays. Moreover, graphical feedback offers comparably lower forces on average than bilateral control at low delays $(0,100$ and $200 \mathrm{~ms})$. This result suggests that graphical feedback, despite taking a longer time for retraction, can maintain lower force interactions than both unilateral and bilateral modalities. For the alignment subtask, neither the graphical mode nor the bilateral mode seemed to affect the normalized force distributions. However, during the insertion subtask, the graphical and bilateral modes helped reduce the average force interactions compared to the unilateral case (Fig. 9c). In contrast with the retraction subtask, tasks performed with graphical feedback exhibited a wider, flatter distribution of forces across all delays. Despite leading to more frequent occurrence of low forces $(|F|<0.4 \mathrm{~N})$ on average than bilateral control, graphical feedback is unable to reduce some of the higher forces $(|F|>0.6 \mathrm{~N})$, suggesting that bilateral control offers a tighter range of low-force interactions, particularly for the insertion subtasks.

\subsection{Maximum Forces}

Often, an important factor in telemanipulation is the maximum slave or environment contact force allowed by the task. We considered the 95 th percentile forces for each 

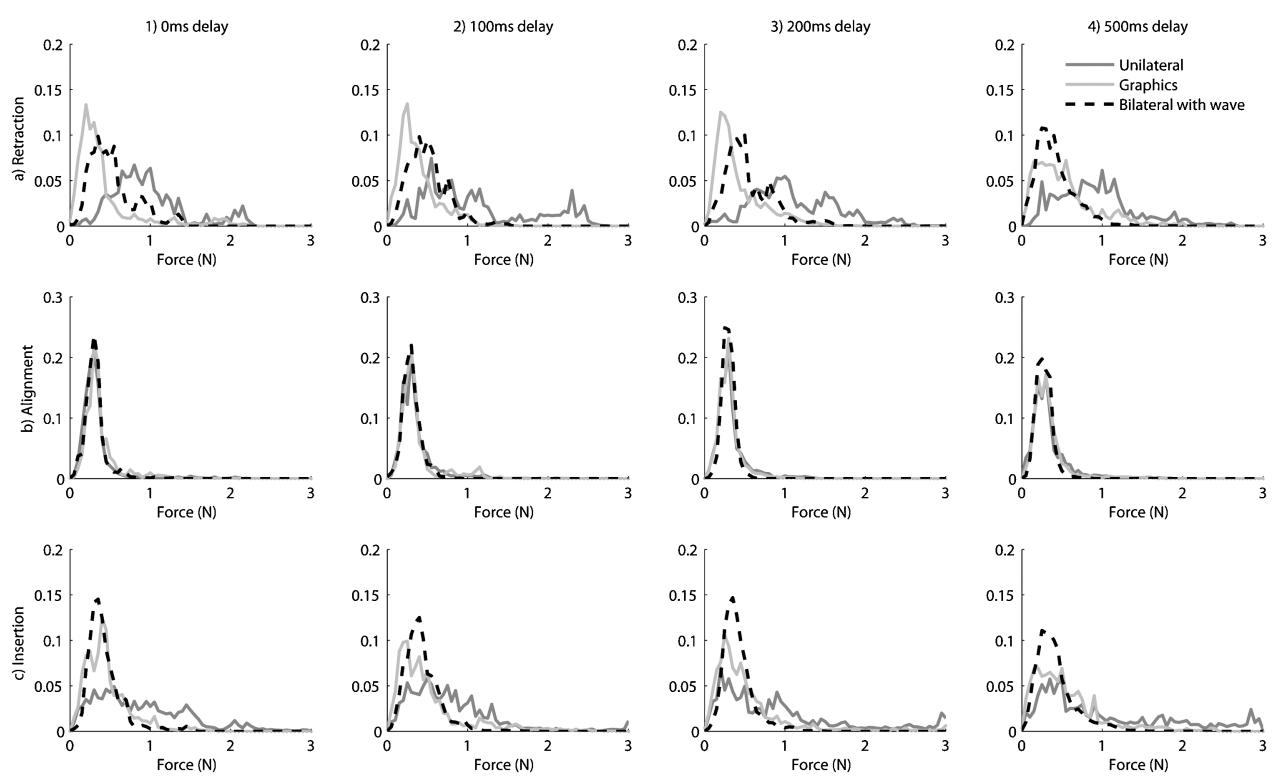

Figure 9. Normalized force histogram distributions across delay.

modality at different delays (Fig. 10a-d). We observe that the maximum forces exerted in unilateral teleoperation were lowered substantially by the introduction of graphical feedback and haptic feedback at all delays (Table 5, $t$-test $p$-values between unilateral and graphical feedback for $0,100,200,500 \mathrm{~ms}$ delay: $1.30 \mathrm{E}-03$, $2.50 \mathrm{E}-04,7.30 \mathrm{E}-0.4,6.60 \mathrm{E}-03$, respectively; $t$-test $p$-values between unilateral and bilateral control for 0, 100, 200, 500 ms delay: 3.38E-04, 1.29E-05, 6.04E-04, 9.78E-04).

Graphical feedback and bilateral control both appear to reduce the maximum forces to approximately the same level at low delays (0 and $100 \mathrm{~ms})$ with greater separation between the two modalities as the delay increases to $500 \mathrm{~ms}$. We also considered the variance of the maximum forces among different feedback modalities (Fig. 10e-h) and found that there is an overall drop in the variance of the maximum forces from the unilateral case when graphical feedback was introduced, and an even more reduction in the variance of forces when bilateral control was introduced. This suggests that, under graphical and bilateral modalities, the maximum forces are generally reduced to a lower level.

A summarized version of the results are given in Table 6, applicable across all delays.

\section{Discussion}

Studies have shown that the ability of human users to perform certain teleoperation tasks is improved when they are provided with haptic feedback. However, when there is a significant delay present in the teleoperation system, the transparency (fi- 

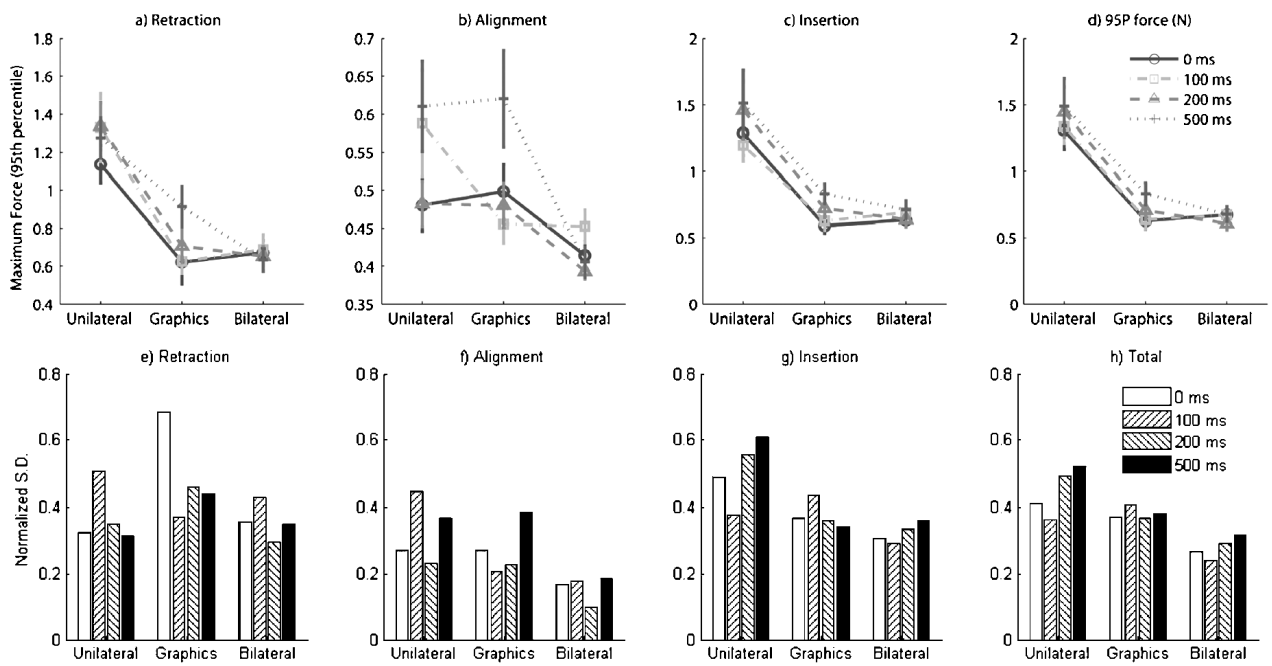

Figure 10. Maximum (95th percentile) forces and corresponding standard deviations across modalities.

delity) of haptic teleoperation is limited due to the adverse effects of stabilizing controllers. Although there have been many studies on how well a teleoperation system performs under delays, there has been surprisingly little work in terms of investigating whether providing haptic feedback is actually beneficial to the performance of a task by the human user. In this paper, we directly measured the performance in a common assembly task under delayed force feedback. This was extended to compare bilateral, graphical and unilateral teleoperation across a range of delays, and we characterized task performance based upon the time required to complete the task and the intensity of force exertions against the environment.

The results show that providing graphical feedback or haptic feedback leads to longer task completion times, regardless of time delay. The difference was only significant during the insertion and extraction subtask, which is consistent with the observation that good coordination of forces is needed to accomplish close-tolerance assembly tasks [19] and that the alignment subtask is largely position-constrained. This result suggests that, given haptic or graphical force feedback, users are more mindful of minimizing forces, which comes at the expense of speed.

We also showed that completion times increase significantly as delay increases for all feedback modalities. Unilateral mode resulted in the least amount of time, followed by bilateral mode, and finally graphical mode. For applications of teleoperation where a certain completion time is desired and higher force interactions can be tolerated, the results suggest that not providing additional feedback (i.e., unilateral control alone) may be sufficient and will result in faster completion times than if force feedback is provided.

Although haptic feedback and graphical feedback increase the completion time of all tasks, we saw that they reduce the applied force, regardless of the time de- 


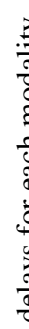

.

巳ัల

ส

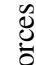

든

()ㅡㄹ

ฮี

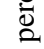

ڤั

声

蔍

ठै

$\stackrel{\text { की }}{\stackrel{0}{*}}$

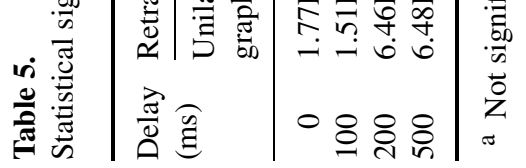

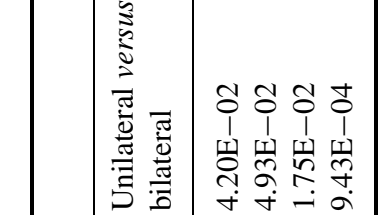

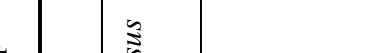

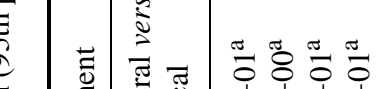

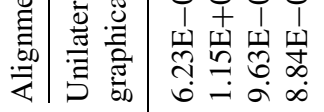

a

我

告

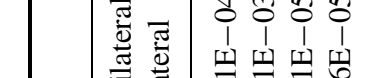

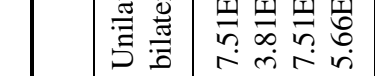

₹

先

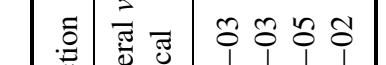

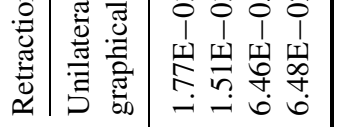
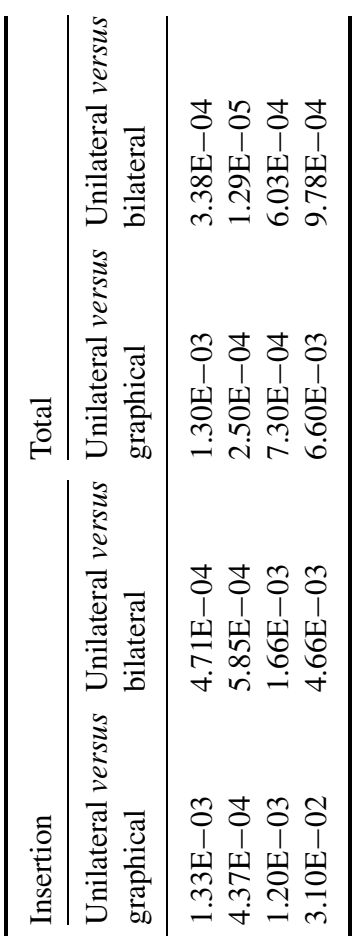

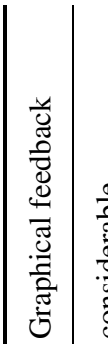

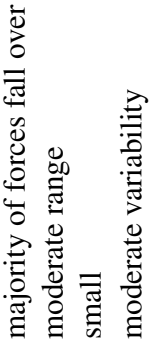

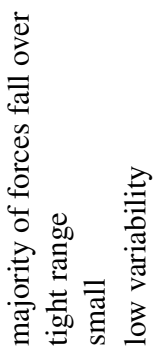

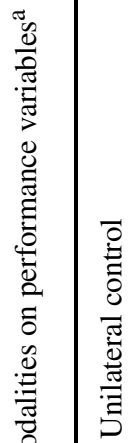

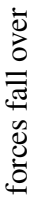

?

to

를

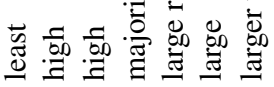

.

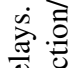

它

它

总

$\because 8$

ב

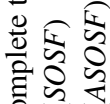

०

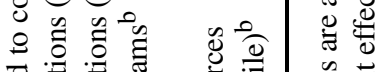

$\cong$

4

远 
lay. In particular, the SOSF and ASOSF plots indicated that haptic feedback could help consistently maintain low forces with low variability even for increasing delays. Graphical feedback originally may seem to result in higher force interactions at increasing delays based on the SOSF plots, but after time-normalizing SOSF measurements, graphical feedback is shown to maintain consistently low force interactions as seen through ASOSF measurements. However, it should be noted that there is less significant reduction in the variance of the SOSF and ASOSF from unilateral control as compared to bilateral control. This suggests that, despite being able to reduce the force interactions, there does not appear to be any reduction in variability of the force interactions.

The force histograms, which depict the frequency of occurrences of the slave/environment contact forces, give a detailed spectrum that helps distinguish exactly during what conditions bilateral and graphical modes offer more desirable force interactions. When considering the normalized force histograms, graphical feedback's highest concentrations of forces settled at a lower value than both the bilateral and unilateral modes for peg retraction. This effect became less pronounced as the delay increased. These results indicate that graphical feedback is able to provide additional information over unilateral and bilateral cases that help users reduce average applied force when the system is at relatively low delay. However, this benefit becomes less pronounced as the delay increases, as the force histograms for graphical feedback begin to diffuse and suggest that it becomes much more difficult to maintain a consistently low force. Despite a similar effect being seen for bilateral control, at higher forces bilateral teleoperation still manages to maintain a relatively high concentration of forces at low levels. When we consider the absolute force histograms, the considerably longer subtask completion times required by graphical feedback result in more forces applied at all levels for nearly all subtasks and delays when compared to bilateral control. Despite the longer completion times required by the graphical and bilateral modes, the unilateral mode still exhibits the largest forces (above $1 \mathrm{~N}$ ). These results were complemented by the maximum (95th percentile) force measurements for each feedback modality, where we observed a significant attenuation of maximum force when provided graphical feedback and when provided haptic feedback.

Our studies have shown that haptic feedback helps for tasks in which minimizing the amount of forces applied to objects and the environment is critical. In addition, we showed that haptic feedback helps minimize the occurrences of larger forces and subjects the task environment to lower forces. However, since the tasks take more time to complete, the lower forces are applied on the environment for a longer period of time. This conflicting relationship between time and force minimization is an apparent trade-off in delayed haptic feedback. We have also shown that graphical feedback helps for tasks in which maintaining lower forces is critical and when there are low delays (below $200 \mathrm{~ms}$ ) in the system; it provides enough information to minimize the range of forces beyond haptic feedback. Again, the cost of increased force reduction is the much longer completion times. Nonetheless, in sit- 
uations where minimizing the forces seen by the environment is a primary concern, graphical feedback (at low delays) and haptic feedback (at any delay) become beneficial; otherwise, unilateral teleoperation may be adequate.

We note that this study is specific to a particular type of manipulation task, i.e., the assembly and disassembly of close-fitting parts. While this is perhaps in the category of tasks where force reflection is clearly beneficial, further studies testing alternative tasks may help to develop an even broader understanding of the benefits of haptic feedback.

This study had to pick an approach for ensuring haptic interaction stability under time delay and we chose the wave variable method. Wave variables were chosen due to their popularity as robust stabilizers for delayed systems; however, there are limitations associated with using wave variables to stabilize a teleoperation system as they tend to sacrifice the transparency of the system in order to ensure a passive communication channel. Despite the limitations of wave variables, we believe that using them as a haptic feedback stabilizer for evaluating task performance under delayed teleoperation is reasonable due to the extensive amount of research on them. Nevertheless, it is interesting to speculate that alternative approaches might have resulted in better task performance given that it is possible to identify the aspects of force feedback that are most important to preserve under delay (still, the relative performance variations across delays are expected to show trends similar to those reported here). On the other hand, if the advantage in terms of system performance offered by any other delay compensation method over the wave-based method that we have used is incremental, the adverse effects of delay on task performance are expected to persist, yielding results similar to those reported in this paper.

In the same respect, this study also employed a particular display of forces (isometric, orthogonal component vectors) to display environment forces. Intuition regarding how to comprehend the Cartesian force display may have played a role in the users' understanding of the environment forces; however, the results were able to indicate that the graphical feedback gave improved force minimization, suggesting that the force information was used properly by the subjects.

The delay range considered in this paper (up to $500 \mathrm{~ms}$ ) is representative of most applications in terrestrial and near-earth environments, e.g., the round-trip time for communication via geostationary satellites is approximately $0.5 \mathrm{~s}$. Larger delays may be encountered in interplanetary space missions and variable delays are relevant for teleoperation over the Internet. Further work will focus on these particular situations.

\section{Conclusions}

In this paper, we considered the benefits of force feedback for task performance in the presence of time delay in the communication channel of a teleoperation system. We evaluated a peg-in-the-hole insertion task through a user study involving unilat- 
eral, bilateral and graphical feedback modalities under different delays. We found consistent and compelling evidence that:

(i) When the users were asked to minimize applied forces, bilateral teleoperation caused the task to take more time compared to unilateral teleoperation, regardless of time delay. Graphical feedback required the most amount of time to complete the task.

(ii) The completion time increased as the delay increased, regardless of teleoperation modality.

(iii) The presence of haptic feedback and the presence of graphical feedback each reduced the environment force, regardless of time delay.

(iv) Haptic feedback helped to minimize the occurrences of high forces regardless of the amount of delay, whereas graphical feedback minimized the occurrences of high forces particularly well for low-delay teleoperation.

These results suggest that for teleoperation systems with considerable delay, the addition of graphical feedback or the addition of haptic feedback is useful when minimizing the forces of contact between the slave robot and its environment is the key requirement. Otherwise, when one is not interested in lowering the slave/environment contact forces, unilateral teleoperation is sufficient and should be considered as it results in faster completion times.

\section{References}

1. B. Hannaford, A design framework for teleoperators with kinesthetic feedback, IEEE Trans. Robotics Automat. 5, 426-434 (1989).

2. D. Lawrence, Stability and transparancy of bidirectional teleoperation, IEEE Trans. Robotics Automat. 9, 624-637 (1993).

3. P. F. Hokayem and M. W. Spong, Bilateral teleoperation: an historical survey, Automatica 42, 2035-2057 (2006).

4. P. Arcara and C. Melchiorri, Control schemes for teleoperation with time delay: a comparative study, Robotics Autonomous Syst. 38, 49-64 (2002).

5. S. Mascolo, Modeling the Internet congestion control using a Smith controller with input shaping, Control Eng. Pract. 14, 425-435 (2006).

6. C. L. Lin, C. H. Chen and H. C. Huang, Stabilizing control of networks with uncertain time varying communication delays, Control Eng. Pract. 16, 56-66 (2008).

7. R. J. Anderson and M. W. Spong, Bilateral control of teleoperators with time delay, IEEE Trans. Automatic Control 34, 494-501 (1989).

8. G. Niemeyer and J. J. E. Slotine, Stable adaptive teleoperation, IEEE J. Oceanic Eng. 16, 152-162 (1991).

9. B. Hannaford and L. Wood, Performance evaluation of a 6 axis high fidelity generalized force reflecting teleoperator, in: Proc. JPL/NASA Conf. on Space Telerobotics, Pasadena, CA, pp. 8997 (1989).

10. G. C. Burdea, Force and Touch Feedback for Virtual Reality. Wiley, New York, NY (1996). 
11. C. Wagner and R. Howe, Force feedback benefit depends on experience in multiple degree of freedom robotic surgery task, IEEE Trans. Robotics 23, 1235-1240 (2007).

12. A. Pressman, L. J. Welty, A. Karniel and F. A. Mussa-Ivaldi, Perception of delayed stiffness, Int. J. Robotics Res. 26, 1191-1203 (2007).

13. H. Ohnishi and K. Mochizuki, Effect of delay of feedback force on perception of elastic force: a psychophysical approach, IEICE Trans. Commun. E90-B, 12-20 (2007).

14. I. Nisky, F. A. Mussa-Ivaldi and A. Karniel, A regression and boundary-crossing-based model for the perception of delayed stiffness, IEEE Trans. Haptics 1, 73-83 (2008).

15. L. N. Verner and A. M. Okamura, Sensor/actuator asymmetries in telemanipulators: implications of partial force feedback, in: Proc. 14th Symp. on Haptic Interfaces for Virtual Environments and Teleoperator Systems, New York, NY, pp. 309-314 (2006).

16. M. J. Massimino, Sensory substitution for force feedback in teleoperation, PhD Thesis, Department of Mechanical Engineering, MIT, Cambridge, MA (1992).

17. M. Kitagawa, D. Dokko, A. M. Okamura and D. D. Yuh, Effect of sensory substitution on suture manipulation forces for robotic surgical systems, J. Thor. Cardiovasc. Surg. 129, 151-158 (2005).

18. B. Hannaford, L. Wood, D. A. McAffee and H. Zak, Performance evaluation of a six-axis generalized force-reflecting teleoperator, IEEE Trans. Syst. Man Cybernet. 21, 620-633 (1991).

19. D. E. Whitney, Quasi-static assembly of compliantly supported rigid parts, J. Dyn. Syst. Meas. Control 104, 65-77 (1982).

\section{Appendix: $t$-Test and ANOVA}

A two-sample Student's $t$-test is a hypothesis test to determine the statistical significance of a pair of data sets, given the assumption that they are independent, standard normal distributions. In particular, $t$-tests determine the confidence level for which the null hypothesis is true.

Given $n$ pairs of $x_{i}$ and $y_{i}$ measured values in a sample population, let $\hat{x}_{i}=$ $\left(x_{i}-\bar{x}\right)$ and $\hat{y}_{i}=\left(y_{i}-\bar{y}\right)$. Then, a $t$ value can be calculated:

$$
t=(\bar{x}-\bar{y}) \sqrt{\frac{n(n-1)}{\sum_{i=1}^{n}(\hat{x}-\hat{y})^{2}}} .
$$

A look-up table of Student's $t$-distribution confidence intervals is then used to determine the probability $p$ that the null hypothesis is true, where the most commonly accepted value for justifying statistical significance is $p=0.05$. The $t$-test is used in this paper to determine whether there is a statistical significance between measured values of different modalities at a certain delay.

ANOVA is a generalization of the two-sample Student's $t$-test. It is a hypothesis test to determine a statistical difference between the means of a dependent variable across different groups. One-way ANOVAs are used when the study involves three or more levels of an independent variable. The ANOVA test produces an $F$-statistic that can then be used to determine the statistical significance ( $p$-value) of the differences of sample means. The use of ANOVA over multiple $t$-tests helps reduce the occurrences of type I error, the error associated with rejecting the null hypothesis when it is actually true. In the case of this paper, ANOVA is used to determine 
whether there is a statistical significance $(p \leqslant 0.05)$ between measured values at different delays for a certain modality.

\section{About the Authors}

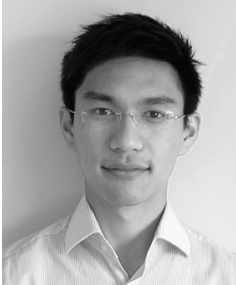

Michael C. Yip received his BASc in Mechatronics Engineering from the University of Waterloo, Canada, in 2009. During his studies, he worked in various industrial and research positions in the field of robotics, sensors and instrumentation, and medical engineering. He is currently working towards a MASc in Electrical Engineering from the University of British Columbia, Canada. His current research interests are in haptics, robotics and control, and the application of computer vision technology in robotics and medicine.

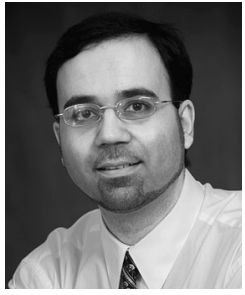

Mahdi Tavakoli received his BS and MS degrees in Electrical Engineering from Ferdowsi University and K. N. Toosi University, Iran, in 1996 and 1999, respectively. He then received his $\mathrm{PhD}$ degree in Electrical and Computer Engineering from the University of Western Ontario, Canada, in 2005. He was a Postdoctoral Research Associate at Canadian Surgical Technologies and Advanced Robotics (CSTAR), Canada, in 2006. He was an NSERC Postdoctoral Fellow with the BioRobotics Laboratory, Harvard University, USA, in 2007-2008. He is now with the Department of Electrical and Computer Engineering at the University of Alberta, Canada. His research focuses on haptics and teleoperation control, medical robotics, and image-guided surgery. He is the Lead Author of Haptics for Teleoperated Surgical Robotic Systems (World Scientific, 2008).

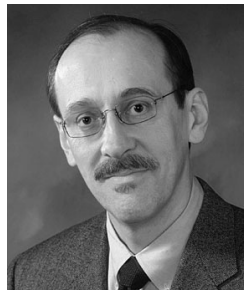

Robert D. Howe is Gordon McKay Professor of Engineering in the Division of Engineering and Applied Sciences at Harvard University. He earned a BA in Physics from Reed College, then worked in the electronics industry as an ana$\log$ and digital design engineer. He earned a $\mathrm{PhD}$ in Mechanical Engineering from Stanford University, in 1990, then joined the faculty of Harvard University. His research interests focus on robot and human manipulation, and the sense of touch. Bioengineering applications include the characterization of the mechanical properties of soft tissues, and the development of new instruments and robotic approaches to minimally invasive surgery. 